\title{
Chromatin diminution leads to rapid evolutionary changes in the organization of the germ line genomes of the parasitic nematodes $A$. suum and $P$. univalens
}

\author{
Christa Bachmann-Waldmann, Stephan Jentsch, Heinz Tobler, Fritz Müller* \\ Departement of Biology, Zoology, University of Fribourg, Chemin du Musée 10, Fribourg CH-1700, Switzerland
}

Received 13 August 2003; received in revised form 4 November 2003; accepted 5 November 2003

\begin{abstract}
Chromatin diminution in the parasitic nematodes Ascaris suum and Parascaris univalens represents a rather complex molecular phenomenon that includes chromosomal breakage, DNA degradation and new telomere formation. At a given elimination site, DNA breakage and new telomere addition does not take place at a single chromosomal locus but at many different places within a several kilobase long chromosomal region, referred to as chromosomal breakage region (CBR). Here we describe the cloning and the characterisation of seven CBRs from A. suum and P. univalens and we show that the process has been conserved between the two species. A detailed sequence comparison provides evidence that the sequences of the CBRs and their flanking regions are not directly important for the specification of the elimination sites. Six out of the seven CBRs are conserved between the two nematode species, suggesting that they have already existed in a common ancestor. We present a hypothesis stating that the elimination process ensures the maintenance of a functional somatic genome and concomitantly allows extremely rapid and profound changes in the germ line genome, thereby allowing the development of new germ line specific functions and thus providing a selective advantage for the chromatin eliminating nematodes during further evolution.
\end{abstract}

Keywords: Ascaris suum; Parascaris univalens; Chromatin diminution; Genome evolution

\section{Introduction}

Chromatin diminution represents an interesting case of a developmentally programmed DNA rearrangement in higher eukaryotes. It happens during mitosis and consists of two separate events, namely of chromosome fragmentation and of the loss of chromosomal material in the prospective somatic cells. Based on cytological observations, chromatin diminution has been reported to occur in 10 different nematode species, most of them belonging to the parasitic family of Ascarididae (reviewed in [1]). In all species, this process takes place during early embryogenesis in all pre-somatic cells concomitantly with soma and germ line segregation and therefore is linked to germ line-soma differentiation.

Abbreviations: agp-1, Ascaris GTPase-like protein; CBR, chromosome breakage region from A. suum; cbr, chromosome breakage region from P. univalens; DIG, Digoxigenin; PCR, polymerase chain reaction

* Corresponding author. Tel.: +41-26-300-8896; fax: +41-26-300-4197.

E-mail address: fritz.Mueller@unifr.ch (F. Müller).
At the cytological and molecular levels, the phenomenon of chromatin diminution has been investigated mainly in the horse intestinal parasite Parascaris univalens and in the related hog intestinal parasite Ascaris suum. The number and the organisation of the germ line chromosomes of the two species are completely different. $P$. univalens carries only two large chromosomes $(2 n=2)$ in the germ line cells. During the process of chromatin diminution the central euchromatic regions of the chromosomes fragment into $2 \times(29 \mathrm{~A}+$ $6 \mathrm{X}$ ) (females) and $2 \times 29 \mathrm{~A}+6 \mathrm{X}$ (males) chromosomes, which segregate individually to the two daughter nuclei [2]. The large heterochromatic ends, however, are rendered to the cytoplasm where they eventually degrade. The germ line genome of A. suum is contained in 19 autosomes and five $\mathrm{X}$ chromosomes $(2 n=38 \mathrm{~A}+10 \mathrm{X}$ in females, $38 \mathrm{~A}+5 \mathrm{X}$ in males). All detectable of them are involved in the elimination process, and elimination results in an increase of the chromosome number to $2 \times(28 \mathrm{~A}+8 \mathrm{X})$ in females and $2 \times 28 \mathrm{~A}+8 \mathrm{X}$ in males in post diminution cells $[1,2]$. Interestingly, despite of the very different size and chromosomal organisation 
in the germ line, genome size and chromosome number of the somatic cells of both species are almost identical $[2,3]$.

Chromatin diminution results in the loss of all detectable heterochromatin from all pre-somatic cells of both nematode species (reviewed in [1]). At the DNA level, about $80-90 \%$ of the total nuclear germ line DNA in P. univalens, and 25\% in A. suum become eliminated [1]. The germ line-specific DNA of both species is highly enriched in repetitive satellite sequences, which are mostly but not completely eliminated from the prospective somatic cells. The eliminated genomic fraction of A. suum also contains middle-repetitive and single copy sequences, among them at least three different single-copy genes, which are lost from the somatic cells of both nematodes (reviewed in [1]).

Little is yet known about the molecular mechanism involved in chromatin diminution. Previously, we have cloned and analysed one somatic telomere (pTel1) from A. suum [4-6]. We found that it was newly formed during the process of chromatin diminution upon chromosomal breakage and addition of 4-6 kb of nematode telomeric repeats (TTAGGC) $n$ to the chromosomal breakage sites [7]. Telomeric sequences do not exist at or near the breakage sites on the germ line chromosome and therefore are added de novo, probably by a developmentally regulated telomerase activity [7]. Interestingly, formation of pTel1 does not take place at a single chromosomal locus, but can occur at many different sites within a specific, several kb long chromosomal region, referred to as chromosomal breakage region 1 (CBR1) [6]. Telomeres are not only attached to the broken ends of the retained chromosomal portions in the pre-somatic cells, where they are most likely required for the maintenance of the chromosomal integrity, but also to the eliminated part of the chromosomes, which later on become degraded in the cytoplasm [5]. Thus, de novo telomere formation during chromatin diminution may represent a non-specific process that can heal any broken DNA end.

At present it is not known how many different CBRs are encoded by the genome of $A$. suum, how they are recognised by the specific trans-acting factors necessary for the elimination process, and how the chromosomes are broken during chromatin diminution. To get further information about these processes we have cloned and analysed two additional CBRs from the genome of A. suum. Furthermore, we have isolated five different CBRs from the genome of $P$. univalens and we show that the mechanism of chromatin diminution is the same in A. suum and P. univalens. Orthologs of six out of the seven analysed CBRs are conserved between the two nematode species, suggesting that they must have existed already in a common ancestor. All CBRs belong to the family of single copy sequences and do not share any homologies with each other. We present evidence that the sequence of the CBRs are not important for their function and discuss alternative models how they might be recognised by the elimination process.

\section{Experimental procedures}

\subsection{Isolation of A. suum and P. univalens genomic DNA}

Adult A. suum and P. univalens worms were collected from infected pigs or horses in a local slaughterhouse. Females were dissected and oocytes, intestines and eggs were collected [8]. For development, eggs were incubated at $30^{\circ} \mathrm{C}$ (A. suum) or $37^{\circ} \mathrm{C}$ (P. univalens) in water containing $0.1 \% \mathrm{H}_{2} \mathrm{SO}_{4}$. Four-cell stage embryos were harvested after $60-72 \mathrm{~h}$ (A. suum) or $4 \mathrm{~h}$ (P. univalens) and larvae after 20-30 days (A. suum) or 4-5 days (P. univalens). The removal of the chitinous layer (peeling) and the DNA isolation was performed as described in $[8,9]$.

\subsection{Somatic A. suum end library}

To clone the chromosomal ends we treated $100 \mu \mathrm{g}$ high molecular weight $A$. suum larval genomic DNA with T4 DNA-polymerase (Roche) for $30 \mathrm{~min}$ at $37^{\circ} \mathrm{C}$ in a buffer containing $50 \mathrm{mM}$ Tris-HCl, $15 \mathrm{mM}$ ammonium sulphate, $7 \mathrm{mM} \mathrm{MgCl} 2,0.1 \mathrm{mM}$ EDTA, $10 \mathrm{mM}$ 2-mercaptoethanol, $33 \mu \mathrm{M}$ dATP, dCTP, dGTP, dTTP, $100 \mu \mathrm{g} / \mathrm{ml}$ bovine serum albumin (BSA), at pH8.5 to make the telomeres blunt ended. The reaction was stopped with $0.11 \mathrm{M}$ EDTA and purified by extraction with phenol/chloroform 1:1 and chloroform/isoamylalcohol 24:1 and precipitated with ethanol. To the blunt ends of chromosomal DNA $2 \mu \mathrm{g}$ of an EcoRI-linker was ligated. The DNA was then phenol extracted and precipitated. After EcoRI digestion and a further phenol extraction step, the EcoRI-linkers were removed by passing the DNA over a Pharmacia S300 spun column. With a $10-40 \%$ sucrose gradient [10] 7-10 kb long DNA fragments were selected and cloned in the EcoRI site of a $\lambda$ ZapII bacteriophage (Stratagene). The unamplified library was screened with an A. suum telomeric (TTAGGC) 27 probe [6]. The library yielded several hundred positive clones of which some were randomly selected and isolated. Bluescript SK (-) plasmids containing the telomeric inserts were excised by following the Stratagene protocol. Plasmid DNA was prepared using standard procedures (Qiagen, [10]).

\subsection{Somatic P. univalens telomere library}

One hundred micrograms of total $P$. univalens larval genomic DNA was partially digested with the enzyme DdeI, which cleaves once in every telomeric repeat TTAGGC. After precipitation, the digested DNA was made blunt ended and EcoRI/NotI adaptors (Stratagene) were ligated to the ends of the fragments. The fragments were then phenolextracted and precipitated. The EcoRI/NotI adaptors were removed by passing the DNA over a Pharmacia S300 spun column. By gel extraction, fragments of $6-10 \mathrm{~kb}$ were isolated and cloned into a $\lambda$ ZapII vector digested with $E c o$ RI (Stratagene). The amplified library was then screened with a random primed DIG-labeled A. suum telomeric 
(TTAGGC) 27 probe [6]. Bluescript SK (-) plasmids containing the telomeric inserts were excised by following the Stratagene protocol. Plasmid DNA was prepared using standard procedures (Qiagen, Promega, [10]).

\subsection{Somatic A. suum subtelomeric probes}

Subtelomeric hybridisation probes from telomeric clones were generated by removing the telomeric repeats by exonuclease III digestion (pTel2 and pTel3) $[10,11]$ or by PCR amplification with DIG-dUTP (ptel7, ptel18, ptel21 and ptel22).

\subsection{P. univalens and A. suum genomic germ line library}

The germ line regions corresponding to the somatic telomeres were isolated by screening a genomic LambdaGEM-12 library from $P$. univalens oocytes and a genomic EMBL3 library from A. suum oocytes made from partially Sau3AI digested genomic $P$. univalens and $A$. suum oocyte DNA. As hybridisation probes we used the somatic subtelomeric probes (see above).

\subsection{Southern blot hybridisation}

Southern blots were hybridised overnight with randomprimed or nick-translated $\left(\alpha{ }^{32} \mathrm{P}\right)$ ATP probes in $40 \%$ formamide, $4 \times$ SSC, $5 \times$ Denhardt's solution, $0.1 \% \mathrm{NaPiPi}$, $0.1 \%$ SDS, $10 \mathrm{mM}$ EDTA, $100 \mu \mathrm{g} / \mathrm{ml}$ sheared herring sperm DNA and $50 \mu \mathrm{g} / \mathrm{ml}$ yeast tRNA at $43^{\circ} \mathrm{C}$ or with probes made by PCR with DIG-dUTP in DIG Easy Hyb (Roche) at $42^{\circ} \mathrm{C}$ (high stringency) and at $37-40^{\circ} \mathrm{C}$ (low stringency). All further steps were performed following the protocol described in the Application Manual by Roche.

\subsection{DNA sequencing and sequence analysis}

Sequencing was performed by the chain termination method with Sequenase 2.0 (U.S. Biochemicals, [12]) or by Microsynth. The sequences were analysed by using the computer programs of the Genetic Computer Group [13], ClustalW at the European Bioinformatics Institute, MEME at the San Diego Supercomputer Center [14], ORF Finder at the National Center for Biotechnology Information, Bethesda MD, Genefinder at the Istituto Tecnologie Biomediche Avanzate, Segrate, Blast programs at the National Center for Biotechnology Information, Bethesda MD, at the Sanger Centre, Cambridge, and at EMBnet-CH/SIB/CSCS, Switzerland.

\subsection{Cloning of telomere addition sites}

Different primers homologous to cbr1, cbr7, cbr18, cbr21 and cbr22 in P. univalens or CBR2 in A. suum and a telomere complementary primer $\left(5^{\prime}\right.$-AAGCCTAAGCCTAAGCC- $\left.3^{\prime}\right)$ were used to amplify the newly formed somatic telomeres by PCR [5]. Best results were obtained by using the Taq DNA polymerase from Perkin Elmer with 30 cycles $\left(95^{\circ} \mathrm{C}, 45 \mathrm{~s}\right.$; $50{ }^{\circ} \mathrm{C}, 1 \mathrm{~min} ; 72^{\circ} \mathrm{C}, 2 \mathrm{~min}$ ) followed by a terminal elongation step $\left(10 \mathrm{~min}\right.$ at $\left.72^{\circ} \mathrm{C}\right)$. For analysing $5 \mu \mathrm{l}$ of a $25 \mu \mathrm{l}$ PCR reaction was loaded on a $1 \%$ agarose gel. The remaining $20 \mu \mathrm{l}$ were precipitated with ethanol. The DNA was then ligated in a Promega T-Vector, amplified in XL1 blue cells and sequenced as described above.

\subsection{Accession numbers}

The sequences of the different CBRs and their surrounding regions are available from GenBank under the accession number AY350753-AY350757 for P. univalens cbr1, cbr7, cbr18, cbr21, and cbr22, AY350751 and AY350752 for A. suum CBR2 and CBR3, AY350758-AY350762 for cbr7, cbr18 and cbr21 orthologs in A. suum, AY350764 for P. univalens rps19s cDNA (partial sequence), AY350763 for P. univalens rps19g cDNA (partial sequence), AY350765 for A. suum genomic sequence rps19s (partial sequence), AY350768 for P. univalens genomic sequence rps19s (partial sequence), AY350766 and AY350767 for P. univalens genomic sequence rps19g (partial sequence).

\section{Results}

\subsection{A. suum somatic telomeres are newly created during chromatin elimination}

We have shown previously that a single cloned somatic telomere, pTel1, is created de novo during the elimination process by chromosomal breakage and addition of several $\mathrm{kb}$ of the telomeric repeat TTAGGC $[1,6,7]$. To assess whether the events observed at pTel1 reflect a general mechanism by which most if not all somatic telomeres are generated, we have analysed additional A. suum somatic telomeres. By screening an A. suum end library with the telomeric probe (TTAGGC) 27 [6], we isolated 13 putative telomeric clones. Partial sequencing revealed that ten of them contained 13-45 perfect repeats of the hexamer TTAGGC at the ends of their inserts. The orientation of the G-rich strand corresponded to the defined orientation of eukaryotic telomeric sequences (for review, see [15]), suggesting that these clones represented cloned telomeres. Two of them (pTel2 and pTel3) were randomly selected for further analysis (see Fig. 1, panel A).

Subtelomeric probes from $\mathrm{pTel} 2$ and $\mathrm{pTel} 3$ were used to isolate the corresponding germ line specific regions ( $\lambda$ ascgl2 and $\lambda$ ascgl3, see Fig. 1) from an A. suum oocyte DNA library. A comparison of the structures of $\lambda \operatorname{ascgl} 2$ and $\lambda$ ascgl3 with those of pTel2 and pTel3 suggested that both telomeres were created during the process of chromatin diminution upon chromosome breakage and new telomere addition (Fig. 1). This was confirmed by Southern blot experiments with different restriction fragments derived from $\lambda \operatorname{ascg} 12$ and $\lambda$ ascgl3. Probe 1 , located proximally to the end 


\section{CBR2}

(A)

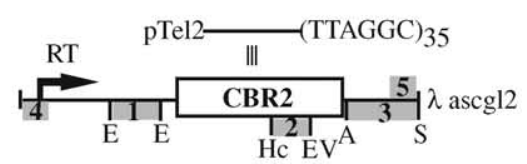

(B)
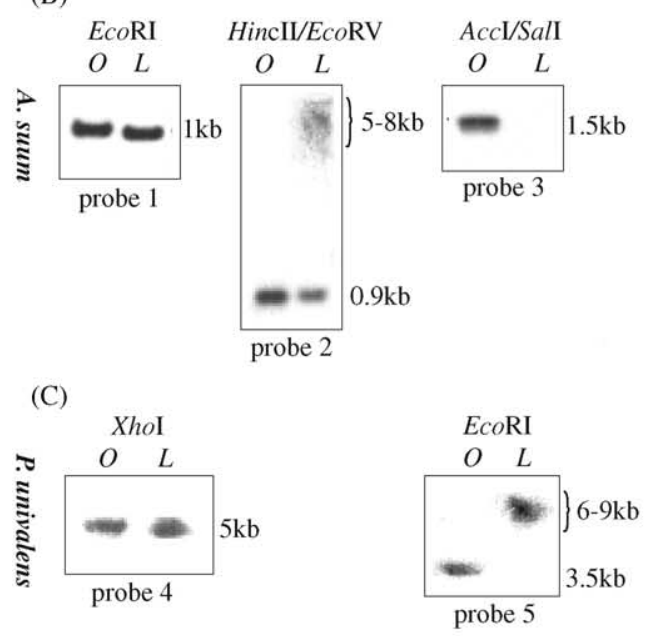

\section{CBR3}

(A)

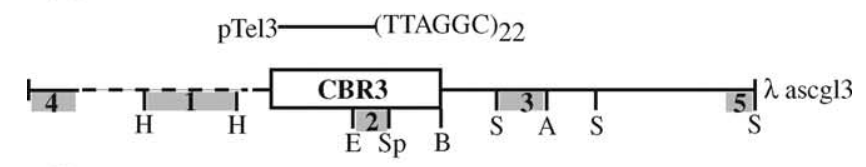

(B)
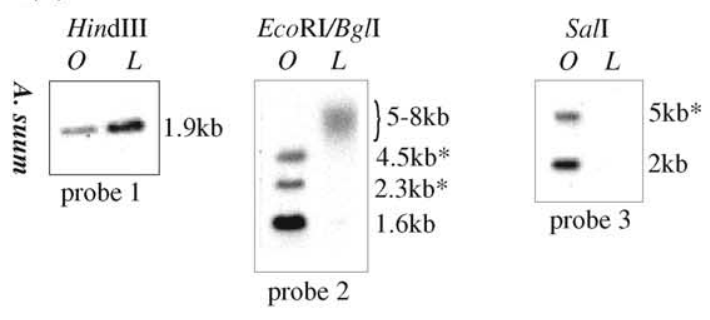

(C)
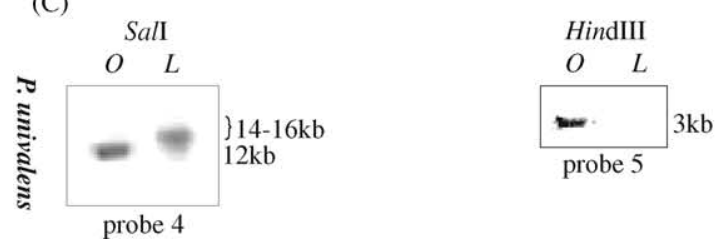

Fig. 1. Somatic telomeres are newly formed upon chromatin diminution in A. suum and in P. univalens. (A) Schematic representation of the two cloned somatic A. suum telomeres pTel2 and pTel3. The number of telomeric repeats contained in these clones is indicated and partial restriction maps of the corresponding germ line chromosomal regions ( $\lambda$ ascgl2 and $\lambda$ ascgl3) are shown. $\lambda$ ascgl2 and $\lambda$ ascgl 3 were completely sequenced (with exception of a fragment in $\lambda$ ascgl3 that is indicated by a dashed line). The non-eliminated regions are on the left, the eliminated regions on the right. Localisation and minimal extension of the two CBRs, as determined by Southern blot and PCR amplification experiments (see text), are indicated with boxes. The positions of three additional PCR amplified of pTel2, annealed to single copy DNA fragments in germ line and somatic DNA (Fig. 1). Probe 3, located distally to the end of the somatic telomere, however, hybridised only to germ line but not to somatic genomic DNA, suggesting that the corresponding genomic region was lost during the process of chromatin diminution (Fig. 1). Probe 2 from $\lambda \operatorname{ascg12}$, that covered the telomeric end of pTel2, light up a $0.9 \mathrm{~kb}$ long single copy band in HincII/EcoRV digested oocyte DNA. The same band, but weaker, together with a faint smear of 5-8 kb long telomeric fragments was present in larval DNA (see Fig. 1). The co-existence of a distinct band with a telomeric smear indicated that chromosomal breakage and new telomere addition at pTel 2 occurred not at a single chromosomal locus, but at different sites within this region. The $0.9 \mathrm{~kb} H i n \mathrm{cII} / E c o \mathrm{RV}$ fragment in the somatic DNA resulted from elimination events where telomere formation occurred in the chromosomal region downstream of the EcoRV site. The existence of multiple telomere addition sites within the chromosomal region of pTel2 was shown independently by performing a telomere amplification experiment by PCR as previously described for CBR1 [5]. By using several forward primers derived from the genomic region of pTel2, a reverse primer complementary to the newly added telomeric repeats TTAGGC and total DNA isolated from A. suum L2 larvae as template, we were able to isolate three further telomere addition sites located within the chromosomal region of pTel2 (see Fig. 1). These results and additional Southern blot experiments with different restriction enzymes (data not shown) allowed to narrow down a genomic region of about $3.5 \mathrm{~kb}$ in which chromosomal breakage and new telomere formation occur at many different sites. We refer to it as chromosomal breakage region 2 (CBR2, Fig. 1). Southern blot experiments performed with

amplified telomere addition sites within CBR2 are shown by vertical black strokes. (1-5) Hybridisation probes. RT indicates a predicted ORF encoding a putative protein with similarities to reverse transcriptases. (B) Southern blot experiments on A. suum DNA. Southern blot experiments were done with total genomic DNA isolated from A. suum oocytes (O) and larvae (L) digested with the restriction enzymes indicated by using radiolabelled probes as mentioned. Probes 1 hybridised to non-eliminated single copy fragments in germ line and somatic DNA. Probes 3 hybridised to eliminated single copy bands in germ line DNA only. Probes 2 hybridised to single copy fragments in germ line DNA and to smears of telomeric fragments in somatic DNA. Asterisks indicate additional fragments that do not correspond to $\lambda$ ascg13. (C) Cross-species hybridisations to $P$. univalens DNA. For both CBRs two probes (4 and 5) cross-hybridised under low stringency conditions to Southern blots containing $P$. univalens genomic DNA isolated from oocytes (O) and larvae (L). Probe 4 of $\lambda$ ascgl 2 identified a non-eliminated $5 \mathrm{~kb}$ single copy fragment in $P$. univalens DNA and probe 5 of $\lambda$ ascgl 2 hybridised to a $3.5 \mathrm{~kb}$ single copy fragment in germ line and a telomeric smear in somatic DNA. Probe 4 of $\lambda$ ascgl 3 cross-hybridised to a $12 \mathrm{~kb}$ fragment in germ line and a smear of telomeric fragments in somatic DNA and probe 5 of $\lambda \operatorname{ascgl} 3$ identified an eliminated single copy fragment. The A. suum CBRs are conserved in the genome of $P$. univalens but the exact position of cbr2 is shifted to the right relative to the DNA sequences. RT: hypothetical reverse transcriptase, A: AccI, B: BglI, E: EcoRI, EV: EcoRV, Hc: HincII, H: HindIII, S: SalI, X: XhoI. 
(A)

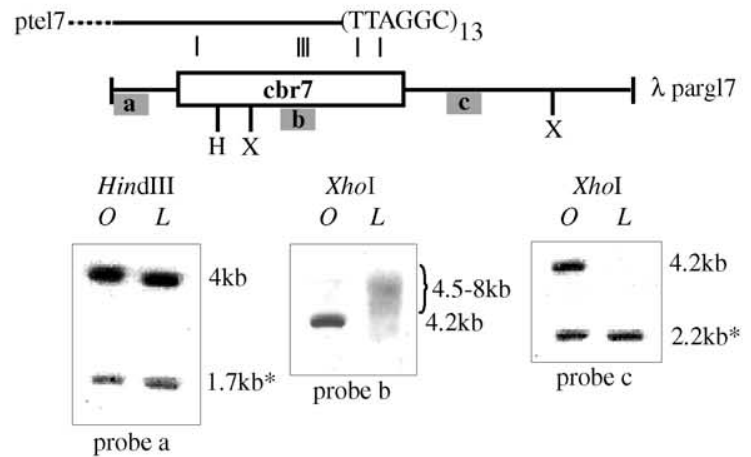

(B)

$\underline{1 \mathrm{~kb}}$
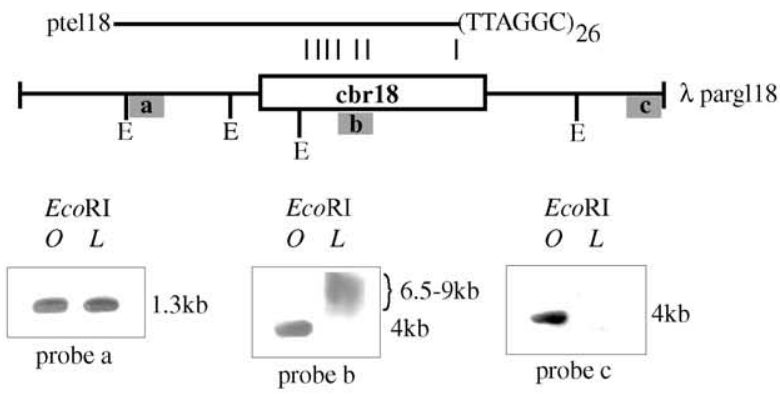

(C)
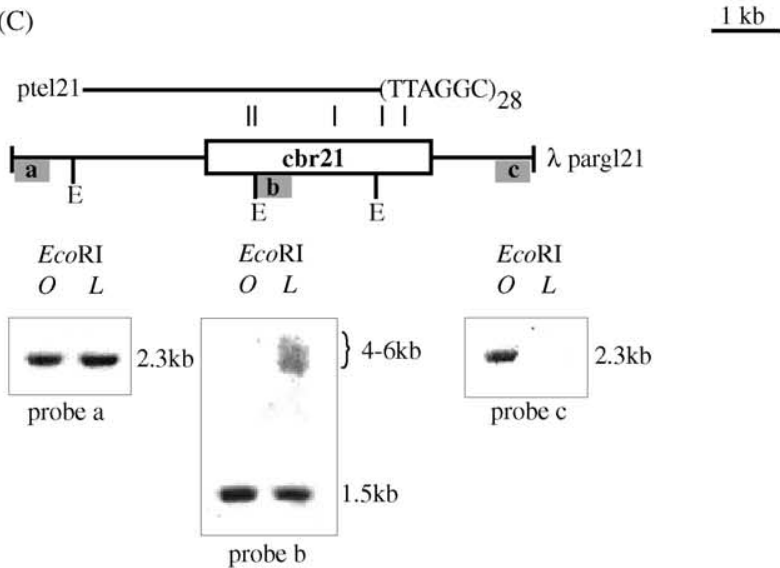

(D)

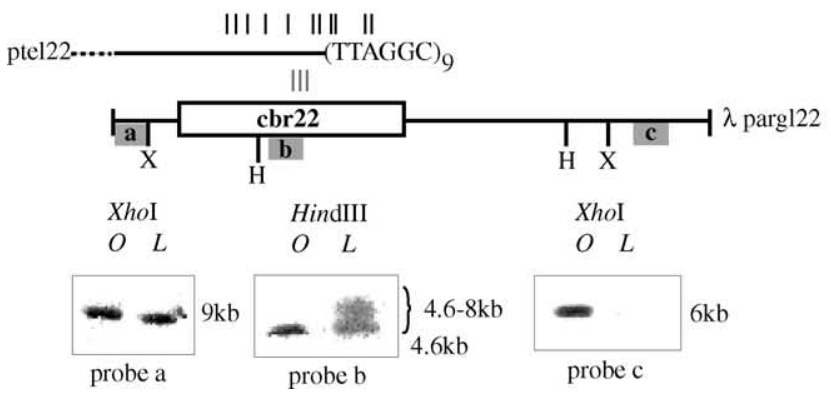

Fig. 2. Somatic telomeres in $P$. univalens are newly formed during chromatin diminution. (A-D) Schematic representation of the four cloned somatic $P$. univalens telomeres ptel7, ptel18, ptel21 and ptel22. The number of telomeric repeats is indicated and partial restriction maps of the corresponding sequenced germ line chromosomal regions ( $\lambda$ pargl7, $\lambda$ pargl18, $\lambda$ pargl21 and $\lambda$ pargl22) are shown. The non-eliminated side of the cbrs
pTel3 yielded similar results (CBR3, see Fig. 1). In summary, these results suggest that the telomere formation mechanism previously described for pTel1 [1,6,7] represents a general mechanism by which the somatic telomeres of $A$. suum are generated during the process of chromatin diminution.

\subsection{The mechanism of chromatin diminution is conserved in P. univalens}

To further extend our knowledge about the elimination process we undertook a phylogenetic analysis of this process in P. univalens. The identification and isolation of sites of chromosomal breakage and new telomere formation was done as described for A. suum. A somatic P. univalens end library was established and screened for telomeric clones with a DIG-labelled (TTAGGC) 27 probe. Seven of about 100 positive clones were randomly selected and partially sequenced. Six of them carried 9-28 telomeric repeats TTAGGC adjacent to the cloning site that were oriented $5^{\prime}$ to $3^{\prime}$ towards the ends of the inserts (Fig. 2 and results not shown). Based on this result we concluded that they represented bona fide telomeric clones and selected four of them (ptel7, ptel18, ptel21 and ptel22) for further analysis (Fig. 2). From a $P$. univalens oocyte genomic library, the corresponding germ line specific DNA regions were isolated $(\lambda \operatorname{parg} 17$ for ptel7, $\lambda$ pargl18 for ptel18, $\lambda$ pargl21 for ptel21 and $\lambda$ pargl22 for ptel22, see Fig. 2). The genomic regions covered by the four $\lambda$ clones extended beyond the ends of the telomeric clones, suggesting that the somatic telomeres were de novo created during the process of chromatin diminution by chromosome breakage and addition of telomeric repeats TTAGGC. This was confirmed by Southern blot experiments with three different probes from each of the four germ line specific regions (Fig. 2). All probes hybridised with the intensity of single copy DNA, indicating that the chromosomal sites were unique and specified particular chromosomal sites. Southern blot hybridisations (Fig. 2) suggested that, like previously demonstrated for A. suum, new telomere addition at the four elimination sites did not occur at a single locus, but took place at many different sites within chromosomal

is on the left, the eliminated side on the right. The extension of the cbrs, as estimated by Southern blot and PCR amplification experiments (see text), is indicated with boxes. The positions of the PCR amplified telomere addition sites to the newly formed ends of the somatic chromosomes are shown by vertical black strokes. The locations of the PCR amplified telomere addition sites to the eliminated DNA of cbr22 are shown by grey strokes. $(\mathrm{a}-\mathrm{c})$ Hybridisation probes. Southern blot experiments were done with total genomic P. univalens DNA isolated from oocytes (O) and larvae (L) that was digested with the restriction enzymes indicated. The filters were hybridised with DIG-labelled DNA probes. Probes a hybridised to single copy fragments present in both, germ line and somatic DNA, whereas probes $c$ hybridised only to the germ line DNA. Probes $\lambda$ parg17a and $\mathrm{c}$ hybridised to additional non-eliminated 1.7 and $2.2 \mathrm{~kb}$ fragments not corresponding to $\lambda$ pargl7 (labelled with asterisks). Probes $\mathrm{b}$ hybridised to a single copy band in germ line DNA and to a telomeric smear in somatic DNA. E: EcoRI, H: HindIII, X: XhoI. 
regions that are a few $\mathrm{kb}$ long (cbrs; for a better distinction, the $P$. univalens cbrs are indicated by small and the A. suum CBRs by capital letters). Indeed, for each of the four cbrs further telomere addition sites were found upon PCR amplification by using total genomic DNA from somatic cells as template (Fig. 2). These results and additional Southern blot experiments (data not shown) allowed an estimation of the minimal size for each of the four cbrs (shown in Fig. 2).

For the A. suum CBR1 we have previously demonstrated that telomeric repeats are not only added to the ends of the somatic chromosomes but also to those of the eliminated chromatin [5]. To test, whether this is also true for P. univalens, a telomere PCR amplification experiment was performed on the eliminated material flanking cbr22. We used several cbr specific primers as described above, but in the opposite orientation, in combination with a telomere complementary primer. As template we took total genomic DNA isolated from synchronised $P$. univalens four-cell stage embryos. At this stage, one or several blastomeres simultaneously undergo chromatin diminution and thereafter contain the eliminated chromatin for a short time before it becomes degraded in the cytoplasm [16]. We were able to isolate three different telomere addition sites (see Fig. 2, cbr22), demonstrating that telomeres are also added to eliminated chromatin. Altogether, our results show that the mechanism of chromatin diminution is highly conserved between $A$. suum and $P$. univalens and occurs in both species at specific CBRs/cbrs.

\subsection{Six out of seven CBRs/cbrs are conserved between the genome of A. suum and P. univalens}

All investigated A. suum and $P$. univalens $\mathrm{CBRs} / \mathrm{cbrs}$ consist of single copy sequences and thus specify unique chromosomal sites. We have tested whether individual CBRs/cbrs are conserved between the genomes of the two nematode species. First, we examined a possible conservation of the A. suum CBR1 in the genome of $P$. univalens. CBR1 is flanked at its non-eliminated side by the highly conserved gene agp- 1 that encodes a putative GTP binding protein [17]. By screening an oocyte genomic library with an A. suum agp- 1 probe we have isolated the unique $P$. univalens agp-1 ortholog that was located on clone $\lambda$ pargl1 (Fig. 3). We established the complete sequence of the $15 \mathrm{~kb}$ long insert of $\lambda$ pargll and compared it to that of the A. suum agp- 1 gene and its flanking regions. The sequence homologies were not restricted to the agp-1 gene, but extended over the entire length of the sequenced regions including the CBR1 region (Fig. 3). However, we noted important differences in the degree of sequence identity. Whereas the agp-1 coding regions had a sequence identity of $>90 \%$, the introns of the agp-1 gene (as far as sequenced) and the CBR1 region shared only $50-80 \%$ of identity (data not

A. suum
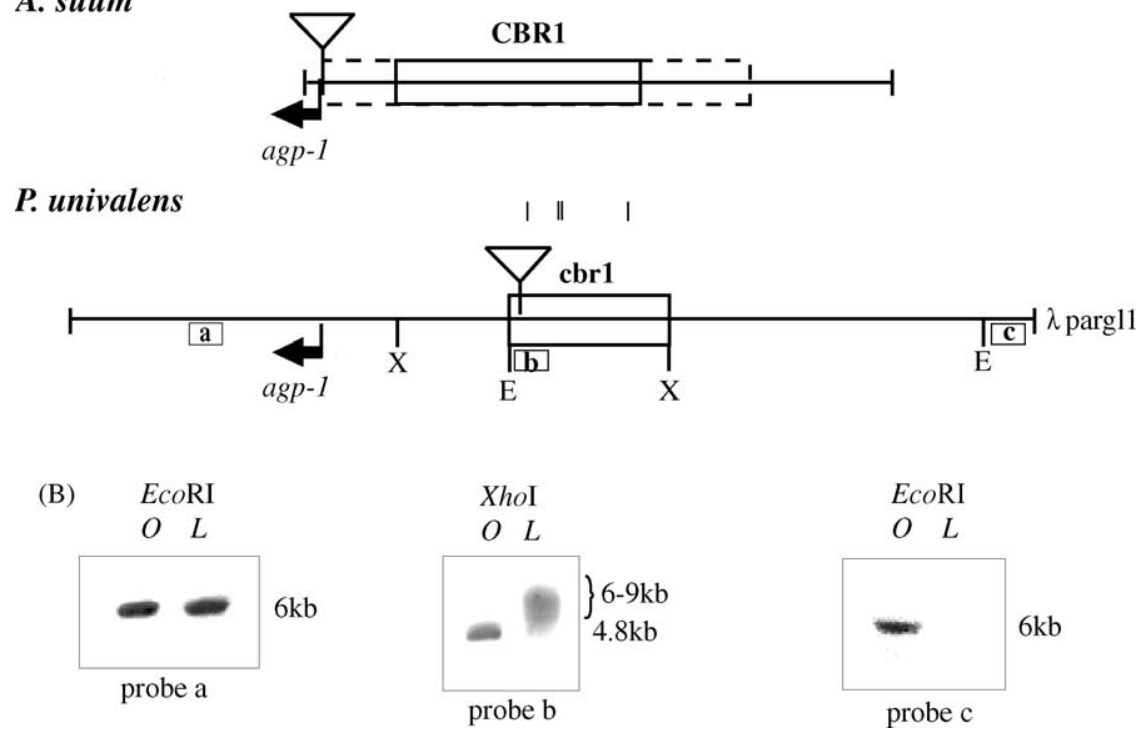

Fig. 3. The A. suum CBR1 is conserved in the genome of $P$. univalens. (A) Schematic representation of the sequenced A. suum CBR1 and the corresponding sequenced $P$. univalens genomic clone $\lambda$ pargl1. A partial restriction map of $\lambda$ pargl1 is shown and the positions of the PCR amplified telomere addition sites are indicated by vertical black strokes. Insertions are shown by triangles. The approximate extension of cbr1 (P. univalens) was determined by Southern blot and PCR amplification experiments (indicated by a box; see text). The extension of CBR1 (A. suum, see 5) as previously determined by Southern blot experiments (indicated by a box; 6) or by PCR amplification experiments (indicated by a dashed box; 5). The non-eliminated regions are on the left, the eliminated on the right. The position of cbr1 is shifted to the right relative to CBR1. (a-c) Hybridisation probes. (B) Southern blot experiments with total genomic P. univalens DNA from oocytes (O) and larvae (L), digested with EcoRI or XhoI, were performed with the DIG-labelled DNA probes indicated. Probe a hybridised to a single copy band present in the germ line specific as well as in somatic DNA. Probe c hybridised to germ line specific DNA only, and probe $\mathrm{b}$ hybridised to a single copy fragment in germ line specific DNA and to a smear of telomeric fragments in somatic DNA. E: EcoRI, X: XhoI. 
shown). Thus, the coding regions of agp-1 are clearly more conserved in evolution than the non-coding introns and the CBR1 region (see discussion). Nevertheless, by performing Southern blot experiments, we found that the $P$. univalens sequence corresponding to CBR1 functions as a site of chromosomal breakage and new telomere formation during chromatin diminution (Fig. 3). The hybridisation data and the results of an independent PCR amplification experiment demonstrated that new telomere addition, like in the A. suum CBR1 and in all other investigated CBRs/cbrs, occurs at many different sites within a chromosomal region of about $3.5 \mathrm{~kb}$ length. We called this region cbr1. Two important differences between the A. suum CBR1 and the P. univalens cbr1, however, were noted: (i) cbr1 contains an insertion of about $1 \mathrm{~kb}$ that is not present in the A. suum ortholog CBR1 (see Fig. 3). This insertion obviously does not interfere with the function of $\operatorname{cbr} 1$ in $P$. univalens, or conversely, its deletion in A. suum has also no adverse effect. (ii) Relative to the orthologous sequence, the position of cbr1 in P. univalens does not match exactly that of CBR1 in A. suum, but seems to be shifted for 1-2 kb (see Fig. 3).

Next, we tested whether the A. suum CBR2 and CBR3 are also conserved in the genome of $P$. univalens. Given the relatively high sequence conservation of $50-80 \%$ between the two CBR1 orthologs, we decided to approach this question by performing a low stringency cross-hybridisation with $A$. suum CBR2 and CBR3 probes on total $P$. univalens genomic DNA. The result is summarised in Fig. 1 (panel C). It shows that the sequences of both CBRs are conserved and function as chromosomal breakage site in the genome of $P$. univalens. As already noted for CBR1, however, the positions of the $P$. univalens $\mathrm{cbr} 2$ and cbr3 seem not match exactly with those of the A. suum orthologs. Rather, they are shifted for a few $\mathrm{kb}$ relative to the same sequences in the two analysed species (Fig. 1).

We then tested whether the $P$. univalens $\operatorname{cbr} 7, \operatorname{cbr} 18, \operatorname{cbr} 21$ and cbr22 are also conserved within the genome of A. suum. Therefore, different probes from each of the four cbrs were cross-hybridised to A. suum genomic DNA under low stringency conditions (Fig. 4, panel B). We found that all probes detected at least one single copy band in the A. suum genomic DNA. The cbr22 derived probes cross-hybridised to a unique region in the genome of $A$. suum with the features typical for a chromosomal breakage region during chromatin diminution (results summarised in Fig. 4). From these data we concluded that not only the sequence but also the function of cbr22 have been conserved in the genomes of $A$. suum and $P$. univalens.

The cbr7 and cbr21 are also conserved in the genome of A. suum. Probe a from cbr7 and probe b from cbr21 hybridised to single copy bands in germ line genomic DNA and to telomeric smears in somatic genomic DNA (Fig. 4). Interestingly, the probes c, located at the eliminated sites of cbr7 and cbr21, cross-hybridised to non-eliminated DNA fragments in the A. suum genomic DNA (see Fig. 4). To further investigate this problem, we have isolated the genomic sequences corresponding to cbr7 and cbr21 from an A. suum oocyte DNA library (cbr7: $\lambda$ ascgl7a with probe a, $\lambda$ ascgl7c with probe $c$; cbr21: $\lambda$ ascgl21a with probe $a, \lambda$ ascgl21c with probe c; see Fig. 4). The analysis of clones $\lambda$ ascgl7a and $\lambda$ ascgl21a confirmed that their sequences encode $A$. suum orthologs of $P$. univalens $\mathrm{cbr} 7$ and cbr21 (called CBR7 and CBR21, see Fig. 4). The homologies between the P. univalens and $A$. suum sequences, however, are restricted to the non-eliminated site and the first third (cbr7) or the first half (cbr21) of the CBRs (shaded boxes in Fig. 4). The rest of the A. suum clones $\lambda$ ascg17a and $\lambda$ ascgl21a show no homology with cbr7 and cbr21. The A. suum sequences corresponding to the eliminated side of cbr7 and cbr21 are contained on the clones $\lambda$ ascg17c and $\lambda$ ascgl21c. These sequences, however, are located elsewhere in the genome of A. suum and represent non-eliminated or only partially eliminated DNA (Fig. 4). In conclusion, the results demonstrate that cbr7 and cbr21 have been maintained in the genome of A. suum, but the eliminated sequences flanking the $A$. suum CBR orthologs have been rearranged. Interestingly, as observed previously for CBR1, CBR2 and CBR3, the exact position of the A. suum homologue of cbr7 relative to the DNA sequence might be slightly shifted compared to that of P. univalens (see Fig. 4).

A sequence homologous to cbr18 is also encoded by the genome of A. suum, but does not function as chromosomal breakage region during the process of chromatin diminution. The cbr18 derived hybridisation probe $\mathrm{b}$ recognised a specific single copy sequence in the genome of $A$. suum that is completely eliminated from all somatic cells (see Fig. 4). A corresponding germ line genomic DNA clone ( $\lambda$ ascgl18) was isolated and entirely sequenced. Sequence comparison revealed that a $2765 \mathrm{bp}$ long DNA segment spanning most of cbr18, was conserved in the genome of A. suum. Outside of this region, the sequences diverged completely between the two nematode species. Thus, the A. suum sequences orthologous to cbr18 are inserted in unrelated single copy DNA sequences (Fig. 4) and become eliminated together with the entire chromosomal region during the process of chromatin diminution. In summary, the sequence of $P$. univalens $\operatorname{cbr} 18$ is only partially conserved in the genome of A. suum and does not function as CBR.

\subsection{No sequence homologies exist between the different CBRs/cbrs}

The Southern blot experiments described above revealed that all CBRs/cbrs analysed here belong to the class of single copy sequences and do not cross-hybridise with each other nor with any other sequences contained in the genome of the two nematode species. To further analyse their structure, we have sequenced the CBRs/cbrs and their flanking regions (see Table 1 for accession numbers). The sequences were submitted to a Blast search, but with the exception of CBR2, they did not share significant homologies to other sequences (Blast) or gene motifs contained in any data base available (Genefinder and ORF Finder). The sequence of 
$\begin{array}{lll}\text { (A) } & 1 \mathrm{~kb} \\ \text { P. univalens } & \text { cbr22 }\end{array}$

(B)

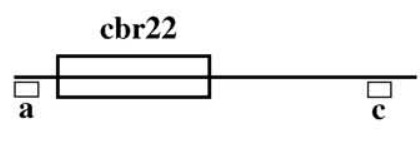

A. suum

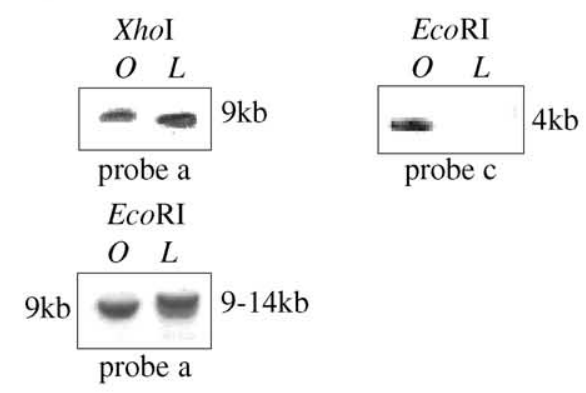

P. univalens

(B)

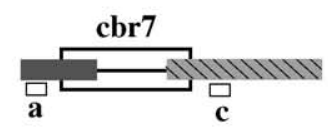

A. suum
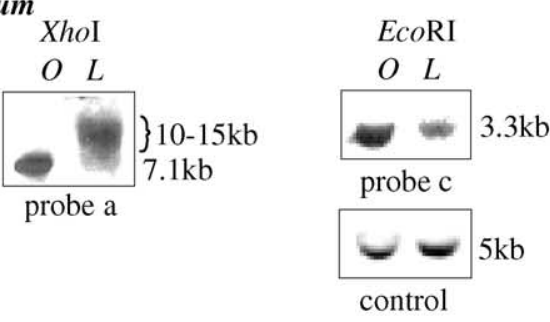

(C)

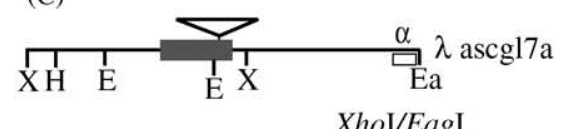

Xhol/EagI
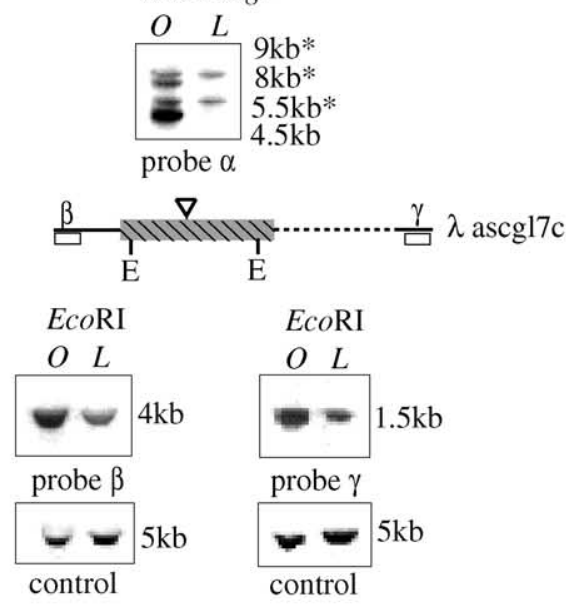

$\underline{1 \mathrm{~kb}}$
(A)

cbr21

$1 \mathrm{~kb}$

\section{P. univalens}

(B)

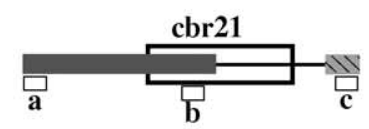

A. suum
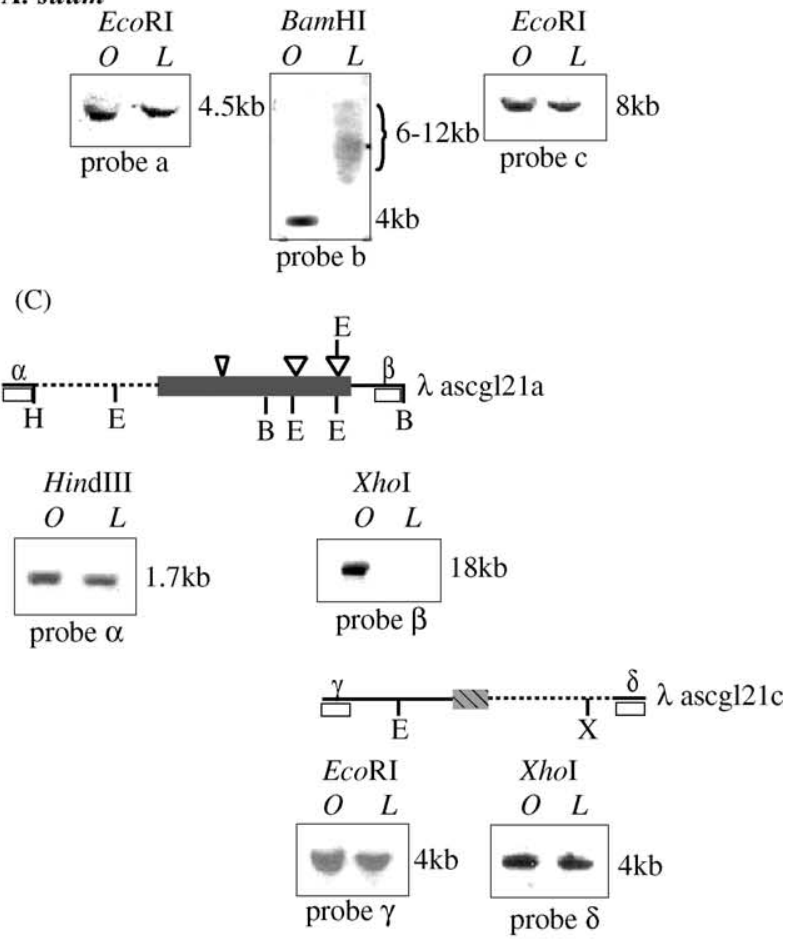

(A)

\section{cbr18}

$\underline{1 \mathrm{~kb}}$

\section{P. univalens}

(B)

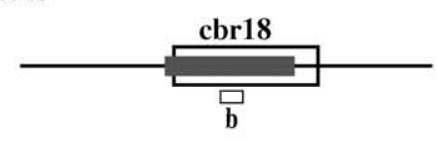

A. suum

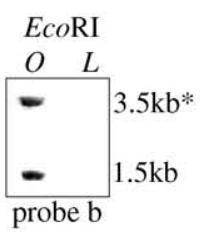

(C)
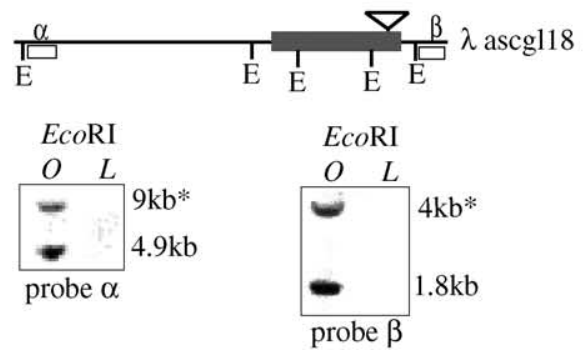
Table 1

\begin{tabular}{llll}
\hline Name & Species & Sequence length (bp) & Accession number \\
\hline cbr1 & P. univalens & 14872 & AY350753 \\
CBR1 & A. suum & 9232 & AY048884 \\
CBR2 & A. suum & 8097 & AY350751 \\
CBR3 & A. suum & 9545 & AY350752 \\
cbr7 & P. univalens & 7516 & AY350754 \\
cbr18 & P. univalens & 9270 & AY350755 \\
cbr21 & P. univalens & 7423 & AY350756 \\
cbr22 & P. univalens & 8660 & AY350757 \\
\hline
\end{tabular}

the A. suum CBR2, however, shares significant homologies to the hypothetical C. elegans gene C34D4.5 that encodes a protein with similarities to reverse transcriptases (Blast) and the Genefinder program did predict a functional gene. The putative reverse transcriptase gene is located at the non-eliminated side of CBR2 (Fig. 1A), in a position similar to that of the previously identified gene agp- 1 that flanks the non-eliminated side of CBR-1 [17], but in opposite orientation.

In order to search for putative short and interspersed signal sequences shared by all CBRs/cbrs that could act as recognition sequence for the elimination machinery, we have submitted their sequences to a computer based sequence analysis program [5]. However, no significant sequence homologies or short interspersed motifs shared by them and their flanking sequences could be found. Par- ticularly, no pre-existing TTAGGC repeats were present in the different CBRs/cbrs. Furthermore, sequence analysis of 36 cloned P. univalens telomere addition sites isolated from five different cbrs (see above) did not reveal any homologies between them. But in all cloned telomere addition sites we found one to six nucleotides that correspond to, and are in frame with, the newly added telomeric repeats (data not shown). This result corresponds to the previously published results from CBR1 in A. suum and argues for a telomerase mediated healing mechanism, in which the free 3 'end of the truncated DNA pairs with the RNA template and initiates the synthesis of new telomeric repeats $[5,7]$.

\section{Discussion}

Here we describe the cloning and analysis of two A. suum and five $P$. univalens somatic telomeres. We show that all of them, like the previously analysed A. suum telomere pTel1 [5,6], become newly created upon chromosomal breakage and new telomere formation during the process of chromatin diminution. Addition of telomeric repeats TTAGGC at a given telomere occurs at many different sites within short, 3.5-7 kb long, specific chromosomal segments called Chromosomal Breakage Regions (CBRs in A. suum or cbrs in $P$. univalens, respectively). Altogether, our results suggest that

Fig. 4. Identification, cloning and characterisation of A. suum genomic regions corresponding to the $P$. univalens cbrs. (A) Schematic representations of the completely sequenced genomic regions containing the P. univalens cbr22, cbr7, cbr21 and cbr18 (see also Fig. 2). (B) Southern blot experiments. Total genomic A. suum DNA from oocytes $(\mathrm{O})$ and larvae $(\mathrm{L})$ was digested with the restriction enzymes indicated and cross-hybridised under low stringency conditions with DIG-labelled DNA probes derived from the P. univalens cbrs as indicated. (C) Schematic representation of the A. suum genomic clones that contain sequences homologous to the $P$. univalens cbrs. Homologous sequences are highlighted by grey bars. The clones are aligned according to their homologies. Insertions are shown by triangles. Southern blot experiments were performed with total genomic A. suum DNA from oocytes (O) and larvae (L) digested with the restriction enzymes indicated. The filters were hybridised with DIG-labelled A. suum probes. Ea: EagI, E: EcoRI, H: HindIII, S: SalI, X: XhoI. cbr22: (A) Schematic representation of cbr22. (a and c) Hybridisation probes. (B) Southern blot hybridisation: Probe a of P. univalens cbr22 cross-hybridised to a non-eliminated single copy fragment in A. suum DNA digested with XhoI or EcoRI and an additional smear of 10-14 kb long telomeric fragments in EcoRI digested larval DNA. Probe c hybridised to a $4 \mathrm{~kb}$ long EcoRI fragment that is absent from A. suum somatic DNA. cbr7: (A) Schematic representation of the completely sequenced genomic region containing the P. univalens cbr7. (a and c) Hybridisation probes. (B) Southern blot hybridisation: Probe a cross-hybridised to a single copy band in the germ line DNA and to a smear of telomeric fragments in larval DNA from A. suum. Probe c recognised a partially eliminated low copy fragment in A. suum genomic DNA. (C) Schematic representation of the sequenced A. suum genomic clones $\lambda$ ascgl7a and $\lambda$ ascgl7c that cross-hybridised with the $P$. univalens probes a and $\mathrm{c}$, respectively (the non-sequenced region of $\lambda$ ascg17c is indicated by a dashed line). $\lambda$ ascgl7a and $\lambda$ ascgl7c do not overlap and represent different loci within the genome of $A$. suum. Sequence homologies between P. univalens and A. suum are indicated with grey and dashed grey bars. An insertion in the sequence of $\lambda$ ascgl7a not present in the sequence of cbr7, is marked with a triangle. $\alpha, \beta, \gamma$ : hybridisation probes. Probe $\alpha$ hybridises to an eliminated $4.5 \mathrm{~kb}$ long single copy band, and faintly to some other bands that are located elsewhere in the genome of A. suum (asterisks). Probes $\beta$ and $\gamma$ hybridise to low copy DNA fragments of which some copies are eliminated from the somatic genome of A. suum. To show that equal amounts of germ line and somatic DNA were loaded, the filters were re-hybridised with probe 4 of $\lambda$ ascg12 (see Fig. 1A). cbr21: (A) Schematic representation of the completely sequenced genomic region containing the $P$. univalens cbr21. (a-c) Hybridisation probes. (B) Southern blot hybridisation: Probes a and c cross-hybridised to non-eliminated single copy bands in A. suum genomic DNA. Probe b recognised a $4 \mathrm{~kb}$ band in oocyte DNA and a telomeric smear of 6-12 kb length in larval DNA. (C) Schematic representation of the sequenced $A$. suum genomic clones $\lambda$ ascgl21a and $\lambda$ ascgl21b that cross-hybridised with the $P$. univalens probes a and $\mathrm{c}$, respectively (the non-sequenced region is indicated by a dashed line). $\lambda$ ascgl21a and $\lambda$ ascgl21b do not overlap and thus represent different loci within the genome of A. suum. Sequence homologies between the sequenced DNA regions from P. univalens and A. suum are indicated with grey bars. Insertions in the sequence of $\lambda$ ascgl21a not present in the sequence of cbr21 are marked with triangles. $\alpha, \beta, \gamma, \delta$ : hybridisation probes. Probe $\alpha$ hybridises to a non-eliminated single copy band, whereas probe $\beta$ recognises an eliminated sequence, thus suggesting that $\lambda$ ascg121a contains a CBR. Probes $\gamma$ and $\delta$ hybridise to non-eliminated single copy sequences. cbr18: (A) Schematic representation of the completely sequenced genomic region containing the $P$. univalens cbr18. (b) hybridisation probe. (B) Southern blot hybridisation: Probe b cross-hybridised to two eliminated bands in A. suum genomic DNA. (C) Schematic representation of the completely sequenced A. suum genomic clone $\lambda$ ascgl18 that cross-hybridised with the $P$. univalens probe b. Sequence homologies between P. univalens and A. suum are indicated with a grey bar. Probes $\alpha$ and $\beta$ identified two eliminated single copy DNA bands in the genome of $A$. suum. Additional bands not corresponding to $\lambda$ ascgl18 are indicated by an asterisk. 
the mechanism of chromatin diminution is highly conserved between the two nematode species.

The CBRs/cbrs and/or their flanking regions are likely to be target sites for specific elimination factors, including DNA-binding proteins and other molecules involved in chromosomal breakage and telomere addition. Therefore, we expected them to contain shared sequence elements or DNA motifs that might serve as recognition signals for the elimination machinery. Instead, we found that all CBRs/cbrs investigated here are composed of single copy sequences and specify unique sites within the genome of the two nematode species. Furthermore, we found that the A. suum and P. univalens $\mathrm{CBR} / \mathrm{cbr}$ orthologs show only a relatively low degree of sequence conservation (between 50 and $80 \%$ of identity), a value comparable to that of non-functional sequences. The non-functional introns of the genes rps19g and rps19s, encoding two small ribosomal proteins S19 [18,19], e.g., have also a sequence identity of $50-80 \%$, whereas the coding regions of these genes share as much as $90 \%$ of identity (results not shown). Thus, the data demonstrate that the sequences of the CBRs/cbrs have not been conserved in evolution between $A$. suum and P. univalens suggesting that their sequences play no role for the elimination process. This conclusion is supported by several other observations: First, the $P$. univalens cbr1 and the A. suum CBR1, CBR7 and CBR21 contain one or several DNA insertions that are not present in the ortholog of the other species (see Figs. 3 and 4). Obviously, insertions (or deletions) can be tolerated without interfering with the elimination process. Second, the orthologs CBR7/cbr7 and CBR21/cbr21 have been rearranged during evolution of the two nematode species, so that their sequences differ after the first third (CBR7/cbr7) or the second half (CBR21/cbr21) of their $\mathrm{CBR} / \mathrm{cbr}$ regions, including the flanking eliminated sequences (see Fig. 4). These chromosomal rearrangements, however, have no detectable influence on the elimination process. Thus, if recognition elements for the elimination machinery exist at the DNA level, they are most likely located at the non-eliminated side of the CBR7/cbr7 and CBR21/cbr21 orthologs. The most compelling evidence against a direct role of the $\mathrm{CBR} / \mathrm{cbr}$ sequences for the elimination process, however, stems from the observation that three of the six CBR/cbr orthologs (CBR1/cbr1, CBR2/cbr2, CBR7/cbr7) did not match exactly to the same DNA sequence within the two nematode species (see Section 3).

Chromatin elimination may not depend on the entire $\mathrm{CBR} / \mathrm{cbr}$ sequences, but on short, interspersed sequence motifs similar to those of prokaryotic restriction enzyme recognition sites. A detailed computer-based sequence analysis, however, did not reveal any evidence for the existence of short homologies or sequence motifs shared between the different CBRs/cbrs and their flanking sequences that could act as recognition signals for the elimination machinery. Furthermore, as demonstrated already for CBR1 $[5,6]$, the analysed CBRs/cbrs contain no pre-existing (TTAGGC) motifs that could specify chromosomal breakage sites and/or telomere addition sites during the elimination process. This is in agreement with the results of earlier experiments showing that new telomere addition during chromatin diminution in A. suum most likely represents a non-specific process mediated by a developmentally regulated telomerase activity that can heal any broken chromosomal end [6]. In summary, the structural data provide no evidence that the sequences of the CBRs/cbrs and their flanking regions themselves may directly be involved in the specification and recognition of the elimination sites by the elimination machinery. We cannot exclude, however, that DNA recognition elements for the elimination machinery could be located outside of the $\mathrm{CBR} / \mathrm{cbr}$ regions and act at distance.

On the other hand, the different CBRs/cbrs may not be recognised by their DNA sequences but through their chromatin structures. Some supporting evidence for this idea comes from the finding that the large germ line chromosomes of $P$. univalens, prior to chromatin diminution, undergo differential mitotic condensation [2]. Condensation occurs only in the regions of the prospective somatic chromosomes, whereas the connecting chromatin (termed chromatin linkers) remains entirely uncondensed. This leads to a 'beads on a string' arrangement of the pre-diminution metaphase chromosomes. During the following anaphase, the somatic chromosomes are released via excision of the linker DNA, which later on is degraded. Similarly, numerous short non-condensed sites interrupt the condensed state in most A. suum chromosomes during the diminution mitosis [2]. Our finding that all known CBRs/cbrs contain both, eliminated and non-eliminated DNA sequences, suggests that they are located at the border between linker DNA and the prospective somatic chromosomes. During the elimination process, they may bind specific proteins that act as a molecular barrier to inhibit chromatin condensation to spread from the region of prospective somatic chromosomes into the non-condensed linker regions. At the same time, the particular chromatin structure of the CBRs/cbrs may act as a recognition signal for the elimination machinery that breaks the chromosomal DNA and adds new telomeres. It was suggested that chromosome breakdown does not occur prior to the onset of chromatid segregation in both species [2]. However, our finding that telomeric repeats are also added to the eliminated DNA and the fact that telomerase is a nuclear enzyme that acts during DNA replication [20] suggest that chromosome breakage and new telomere addition may occur already during the S-phase preceding the elimination mitosis. Later on during the progressing cell cycle, the newly created somatic chromosomes may condense individually, but be kept together by specific "diminution complexes" at their ends so that they accumulate as seemingly intact chromosomes in the equatorial plate during metaphase. During the following anaphase, the somatic chromosomes may then be separated from the eliminated material by segregation forces. Interestingly, telomeric end-to-end association occurs during A. suum spermatogenesis, whereby in the anaphase of both meiotic divisions all 19 autosomes aggregate to form an irregular ring structure that proceeds pole 
wards $[21,22]$. So far, such a model is purely hypothetical and needs to be further investigated. Unfortunately, the functional analysis of the CBRs/cbrs is hampered by the parasitic life style of $A$. suum and $P$. univalens that does not allow genetic analyses. Moreover, so far it was not possible to establish an in vitro system for nematodes that would allow to study the elimination process at the biochemical level.

A further important finding of this work is that six out of the seven investigated CBRs/cbrs are conserved in the genomes of A. suum and P. univalens. This suggests that they have existed already in a common ancestor and that most of them have been conserved throughout evolution. Interestingly, not only the CBRs/cbrs but also the size and the organisation of the somatic genomes [3] have been conserved between the two nematode species, whereas their germ line genomes were subject to a variety of evolutionary changes. These changes include chromosomal rearrangements, amplification and accumulation of large quantities of highly repetitive satellite DNA, and partial genome duplications, altogether resulting in dramatic differences in the size and the chromosomal organisation of the germ line genomes of A. suum and P. univalens (reviewed in [3]). Rapid evolutionary changes in the germ line of these parasitic nematodes are also manifested between the morphologically almost indistinguishable Parascaris varieties, one with a single chromosome and only terminal heterochromatin (P. univalens) and the other one with two chromosome pairs and intercalary heterochromatin (P. equorum) in their germ lines [23]. One would expect that such large-scale rearrangements should have a deleterious effect on the fitness of the organism due to disturbance of the genetic balance. In the cells of the germ line, however, in which gene activity is largely or even completely repressed during many stages of the life cycle, except during germ cell maturation, there may have been little, if any, need for gene dosage compensation. In the somatic cells of every new generation, on the other hand, the highly conserved mechanism of chromatin diminution restores the initial somatic gene balance that is required for normal development. Rapid evolutionary changes in the genomes, therefore, must be restricted to the eliminated material since the non-eliminated material, that is rendered to and functions in the somatic cell lineages, is not expected to be able to accumulate large scale changes. It is interesting to note that the orthologs of the three investigated $P$. univalens cbrs, cbr7, cbr22, and cbr18, are rearranged in the genome of A. suum. This suggests that the CBRs/cbrs may represent "recombinational hot spots" and can easily be rearranged during evolution, in most cases without loosing their function during the elimination process. Thus, the elimination process may ensure the maintenance of a working somatic genome and at the same time allow extremely rapid and profound evolutionarily changes in the organisation of the germ line, thereby leading to the development of new germ line specific functions that provide the chromatin-eliminating nematodes with selective advantages during further evolution
$[8,18,19]$. This may have been particularly important for this class of organisms, which during evolution have adopted a parasitic mode of life requiring the production of an enormous number of eggs to ensure the maintenance of the species.

\section{Acknowledgements}

We thank Yolande Molleyres and Laurence Bulliard for technical help. This work was supported by the Swiss National Science Funds 31-40776.94 and 31-56953.99.

\section{References}

[1] Müller F, Tobler H. Chromatin diminution in the parasitic nematodes Ascaris suum and Parascaris univalens. Int J Parasitol 2000;30:3919.

[2] Niedermaier J, Moritz KB. Organisation and dynamics of satellite and telomere DNAs in Ascaris: implications for formation and programmed breakdown of compound chromosomes. Chromosoma 2000;109:439-52.

[3] Tobler H. The differentiation of germ line and somatic cell lines in nematodes. In: Henning $\mathrm{W}$, editor. Germ line-soma differentiation results and problems in cell differentiation, vol. 13. Berlin: Springer-Verlag; 1986. p. 1-69.

[4] Müller F. New telomere formation during the process of chromatin diminution in the parasitic nematodes A. suum and $P$. univalens. In: Molecular biology intelligence unit 22: telomerases, telomeres and cancer. Georgetown, Texas (USA): Landes Bioscience; 2002. p. 291-9.

[5] Jentsch S, Tobler H, Müller F. New telomere formation during the process of chromatin diminution in Ascaris suum. Int $\mathrm{J}$ Dev Biol 2002;46:143-8.

[6] Müller F, Wicky C, Spicher A, Tobler H. New telomere formation after developmentally regulated chromosomal breakage during the process of chromatin diminution in Ascaris lumbricoides. Cell 1991;18:815-22.

[7] Magnenat L, Tobler H, Müller F. Developmentally regulated telomerase activity is correlated with chromosomal healing during chromatin diminution in Ascaris suum. Mol Cell Biol 1999;19:345765.

[8] Spicher A, Etter A, Bernard V, Tobler H, Müller F. Extremely stable transcripts may compensate for the elimination of the gene fert-1 from all Ascaris lumbricoides somatic cells. Dev Biol 1994;164:7286.

[9] Müller F, Walker P, Aeby P, Neuhaus H, Felder H, Back E, et al. Nucleotide sequence of satellite DNA contained in the eliminated genome of Ascaris lumbricoides. Nucleic Acids Res 1982;10:4937510 .

[10] Sambrook J, Fritsch EF, Maniatis T. Molecular cloning: a laboratory manual. 2nd ed. Cold Spring Harbor, NY: Cold Spring Harbor Laboratory Press; 1989.

[11] Rogers SG, Weiss B. Exonuclease III of Escherichia coli K-12, an AP endonuclease. Methods Enzymol 1980;65:201-11.

[12] Sanger F, Nicklen S, Coulson AR. DNA sequencing with chain-terminating inhibitors. Proc Natl Acad Sci USA 1977;74:54637.

[13] Devereux J, Haeberli P, Smithies O. A comprehensive set of sequence analysis programs for the VAX. Nucleic Acids Res 1984;12: 387-95.

[14] Timothy L, Elkan B, Elkan C. Fitting a mixture model by expectation maximization to discover motifs in biopolymers. In: Proceedings 
of the second international conference on intelligent systems for molecular biology. Menlo Park, California: AAAI Press; 1994. p. 28 36.

[15] Blackburn EH. Telomeres. Trends Biochem Sci 1991;16:378-81.

[16] Esteban MR, Giovinazzo G, Goday C. Chromatin diminution is strictly correlated to somatic behaviour in early development of the nematode Parascaris univalens. J Cell Sci 1995;108:2393-404.

[17] Huang YJ, Stoffel R, Tobler H, Müller F. A newly formed telomere in Ascaris suum does not exert a telomere position effect on a nearby gene. Mol Cell Biol 1996;16:130-4.

[18] Etter A, Aboutanos M, Tobler H, Müller F. Eliminated chromatin of Ascaris contains a gene that encodes a putative ribosomal protein. Proc Natl Acad Sci USA 1990;88:1593-6.
[19] Etter A, Bernard V, Kenzelmann M, Tobler H, Müller F. Ribosomal heterogeneity from chromatin diminution in Ascaris lumbricoides. Science 1994;265:954-6.

[20] Marcand S, Brevet V, Mann C, Gilson E. Cell cycle restriction of telomere elongation. Curr Biol 2000;10:487-90.

[21] Edwards CL. The idiosomes in Ascaris megalocephala and Ascaris lumbricoides. Archiv für Zellforschung 1910;5:422-9.

[22] Felder H. Lokalisierung von hochrepetitiven DNA-Sequenzen und ribosomalen Genen auf Chromosomen von Ascaris lumbricoides var. suum mittels in situ Hybridisierungsexperimenten, diploma thesis; 1983.

[23] Goday C, Pimpinelli S. Chromosome organisation and heterochromatin elimination in Parascaris. Science 1984;224:411-3. 